\title{
Covariance of the redshift-space matter power spectrum after reconstruction
}

\author{
Chiaki Hikage* \\ Kavli Institute for the Physics and Mathematics of the Universe (Kavli IPMU, WPI), University of Tokyo, \\ 5-1-5 Kashiwanoha, Kashiwa, Chiba 277-8583, Japan \\ Ryuichi Takahashi \\ Faculty of Science and Technology, Hirosaki University, \\ 3 Bunkyo-cho, Hirosaki, Aomori 036-8588, Japan \\ Kazuya Koyama \\ Institute of Cosmology and Gravitation, University of Portsmouth, Portsmouth PO1 3FX, United Kingdom
}

(Received 28 July 2020; accepted 22 September 2020; published 9 October 2020)

\begin{abstract}
We explore the covariance of redshift-space matter power spectra after a standard density-field reconstruction. We derive perturbative formula of the covariance at the tree-level order and find that the amplitude of the off-diagonal components from the trispectrum decreases by reconstruction. Using a large set of $N$-body simulations, we also find the similar reduction of the off-diagonal components of the covariance and thereby the signal-to-noise ratio $(\mathrm{S} / \mathrm{N})$ of the postreconstructed (postrec) power spectra significantly increases compared to the prereconstructed spectra. This indicates that the information leaking to higher-order statistics come back to the two-point statistics by reconstruction. Interestingly, the postrec spectra have higher $\mathrm{S} / \mathrm{N}$ than the linear spectrum with Gaussian covariance when the scale of reconstruction characterized with the smoothing scale of the shift field is below $\sim 10 \mathrm{~h}^{-1} \mathrm{Mpc}$ where the trispectrum becomes negative. We demonstrate that the error of the growth rate estimated from the monopole and quadrupole components of the redshift-space matter power spectra significantly improves by reconstruction. We also find a similar improvement of the growth rate even when taking into account the supersample covariance, while the reconstruction cannot correct for the field variation of the supersample modes.
\end{abstract}

DOI: $10.1103 /$ PhysRevD.102.083514

\section{INTRODUCTION}

A biggest challenge in the modern cosmology is the mystery of dark matter and dark energy [e.g., 1]. Largescale structure traced by galaxies is one of the powerful cosmological probes to study the properties of the dark components. The baryonic acoustic oscillation (BAO) imprinted on galaxy distributions is a powerful cosmological probe to study the expansion history of the Universe [2-24]. Bulk motion of galaxies associated with the growth of the large-scale structure generates the anisotropy in the redshift-space galaxy distribution, i.e., the redshift-space distortion, which probes the growth rate of the large-scale structure and is useful to test general relativity and modified gravity models [e.g., 25-31]. The full shape of the power spectrum also has fruitful information of cosmology [e.g., 32]. Upcoming spectroscopic galaxy surveys such as PFS [33], DESI [34], HETDEX [35], Euclid [36], and WFIRST

*chiaki.hikage@ipmu.jp
[37] are expected to do precise cosmological studies to clarify the nature of dark matter and dark energy.

In the linear perturbation theory, different wavelength modes of the fluctuations of matter density field grow independently. Since the gravitational growth of the largescale structure is a nonlinear process, different modes are coupled with each other, which makes a precise cosmological analysis difficult. For example, the BAO signal in the large-scale structure is degraded and the BAO scale is biased at later times [e.g., 38]. The perturbation theory breaks down in the nonlinear regime and thus precise analytical prediction is difficult at small scale. The twopoint statistics such as the power spectrum characterize the whole statistical properties in Gaussian fields. However, non-Gaussianity increases in the evolved matter density field and thereby higher-order moments/correlation functions also become important. The information content of the power spectrum is shown to be saturated on nonlinear scale [39-41]. This indicates that the cosmological information leaks to higher-order statistics beyond two-point 
statistics, which makes our cosmological analysis more complicated.

Density-field reconstruction aims for recovering the initial or linearly evolved density field. A standard BAO reconstruction method proposed by [42] shifts mass particles or galaxies toward their initial Lagrangian positions to recover the original BAO signal. The shift field is estimated using the inverse Zeldovich approximation [43] from the observed (evolved) density field after smoothing small-scale power. The reconstruction effectively undoes the bulk motion and thereby the BAO signal is successfully recovered [44-48]. The BAO reconstruction method has been applied to various galaxy surveys [18-24,49]. It was also shown that the correlation of the reconstructed matter density field with the initial density field extends to more nonlinear scale [47,50-56]. Reference [57] derived the one-loop perturbative formula of the reconstructed matter power spectrum in real space. They found that the amplitudes of the one-loop nonlinear terms decrease substantially by reconstruction and the perturbation theory works at more nonlinear scale. The authors of Ref. [58] extended their formula to redshiftspace clustering and demonstrated that the growth rate measurement from the redshift-space distortion is significantly improved.

How does the reconstruction alter the covariance of the matter power spectra? The covariance of matter power spectra without reconstruction has been investigated both from the perturbation theory and from $\mathrm{N}$-body simulations. The tree-level perturbative formula of the covariance of the matter power spectra was derived to show that the nonGaussian effect generates the correlations between different bands and thereby the covariance has nonzero off-diagonal components [e.g., 3,39]. The matter covariance has been also investigated from a large set of $\mathrm{N}$-body simulations to show that the non-Gaussian effects significantly suppresses the signal-to-noise ratio $(\mathrm{S} / \mathrm{N})$ of the power spectrum and thereby degrades the information content of the power spectrum [40,59-62]. The non-Gaussian effects on the matter covariance come from the mode coupling associated with the nonlinear gravity. Since the reconstruction effectively linearizes the field by partially removing the modecoupling effect, it is expected that the covariance of the power spectrum is more diagonalized.

Here we first investigate the covariance of the redshiftspace matter power spectra after the field is reconstructed and evaluate the information content of the matter power spectrum by using the perturbation theory and a large set of $N$-body simulations. The mode coupling between the small-scale modes and the large-scale modes beyond survey size has a significant contribution to the matter covariance, which is known as "beat coupling" [63] or "supersample covariance (SSC)" [64,65]. We also investigate the effect of SSC on the reconstructed covariance. Finally, we show the improvement of the growth rate measurement from the redshift-space power spectra when using the covariance matrix of reconstructed spectra.

This paper is organized as follows: in Sec. II, we derive a tree-level perturbative formula of the covariance of monopole and quadrupole components of matter power spectra after reconstructing the field. We see how the off-diagonal components of their covariance are changed by reconstruction with different smoothing scales. In Sec. III, we also numerically estimate the covariance using a large set of $\mathrm{N}$-body simulations to see the behavior of the covariance of reconstructed spectra. In Sec. IV, we evaluate the S/N of the reconstructed power spectra to discuss how much the information content is recovered. In Sec. V, we study the impact on the growth rate measurement by using the covariance of reconstructed spectra. We also study the effect of the supersample covariance on our results in Sec. VI. Section VII is devoted to the summary and conclusions.

Throughout this paper, we assume a flat $\Lambda \mathrm{CDM}$ model with the best-fit values of Planck TT, TE, EE + lowP in 2015, i.e., $\Omega_{b}=0.0492, \Omega_{m}=0.3156, h=0.6727, n_{s}=$ 0.9645 , and $\sigma_{8}=0.831$ [66].

\section{PERTURBATIVE FORMULA OF COVARIANCE OF MATTER POWER SPECTRA IN REDSHIFT SPACE}

In this section, we derive the covariance of the monopole $(\ell=0)$ and quadrupole $(\ell=2)$ components of the redshift-space matter power spectrum in a perturbative approach. Our formula is applicable to higher-order multipoles such as hexadecapole $(\ell=4)$; however, we do not include them because their signal-to-noise ratios are small relative to the monopole and the quadrupole and also their signals are dominated by the nonlinear redshift-space distortion.

The covariance can be generally decomposed into the Gaussian and the non-Gaussian parts as

$$
\operatorname{Cov}=\operatorname{Cov}^{(\mathrm{G})}+\operatorname{Cov}^{(\mathrm{NG})} .
$$

When neglecting the convolution with the survey geometry, the Gaussian part is given by

$$
\begin{aligned}
\operatorname{Cov}_{\ell \ell^{\prime}}^{(\mathrm{G})}\left(k_{i}, k_{j}\right)= & \frac{(2 \ell+1)\left(2 \ell^{\prime}+1\right)}{2} \int_{-1}^{1} d \mu \mathcal{L}_{\ell}\left(\mu_{\mathbf{k}_{i}}\right) \mathcal{L}_{\ell^{\prime}}\left(\mu_{\mathbf{k}_{j}}\right) \\
& \times \frac{2}{V} \frac{(2 \pi)^{3}}{V_{k_{i}}} \delta_{i j}^{\mathrm{K}}\left[P^{\mathrm{z}}\left(\mathbf{k}_{i}\right)+\frac{1}{n}\right]\left[P^{\mathrm{z}}\left(\mathbf{k}_{j}\right)+\frac{1}{n}\right],
\end{aligned}
$$

where $\mu_{\mathbf{k}}$ is the cosine angle between $\mathbf{k}$ and the line-ofsight direction, $\mathcal{L}_{\ell}$ is the $\ell$ th Legendre polynomial, i.e., $\mathcal{L}_{0}=1$ and $\mathcal{L}_{2}=\left(3 \mu^{2}-1\right) / 2, V$ is the sample volume, and $n$ is the number density of mass particles. The volume of $k$-binning shell $V_{k_{i}}$ is approximated as $4 \pi k_{i}^{2} \Delta k$ where the 
binning width $\Delta k$ is much smaller the mean wave number of $i$ th bin $k_{i}$. The redshift-space linear matter power spectrum $P^{\mathrm{z}}(\mathbf{k})$ is given by

$$
P^{\mathrm{Z}}(\mathbf{k})=Z_{1}(\mathbf{k})^{2} P_{\mathrm{L}}(k),
$$

where $P_{\mathrm{L}}(k)$ is the linear matter power spectrum in real space and $Z_{1}$ is the first-order Eulerian kernel in redshift space,

$$
Z_{1}(\mathbf{k})=1+f \mu_{\mathbf{k}}^{2}
$$

with the linear growth rate $f \equiv d \ln D / d \ln a$ defined as the logarithmic derivative of the linear growth factor.

Next, we derive the non-Gaussian covariance at tree level. We do not take into account the higher-order covariance such as the one-loop covariance for simplicity. The one-loop covariance becomes important at higher $k$; however, the shot noise usually dominates the error at higher $k$ in actual observed data. In Sec. VI, we take into account the SSC.

The tree-level covariance comes from the tree-level term of the trispectrum of matter power spectra given by [67]

$$
\begin{aligned}
T^{(\text {tree })}\left(\mathbf{k}_{\mathbf{1}}, \mathbf{k}_{\mathbf{2}}, \mathbf{k}_{\mathbf{3}}, \mathbf{k}_{\mathbf{4}}\right)= & 4\left[Z_{2}\left(\mathbf{k}_{\mathbf{1 2}},-\mathbf{k}_{\mathbf{1}}\right) Z_{2}\left(\mathbf{k}_{\mathbf{1 2}}, \mathbf{k}_{\mathbf{3}}\right) P_{\mathrm{L}}\left(k_{12}\right) Z_{1}\left(k_{1}\right) P_{\mathrm{L}}\left(k_{1}\right) Z_{1}\left(k_{3}\right) P_{\mathrm{L}}\left(k_{3}\right)+(11 \text { perms. })\right] \\
& \left.+6\left[Z_{3}\left(\mathbf{k}_{\mathbf{1}}, \mathbf{k}_{\mathbf{2}}, \mathbf{k}_{\mathbf{3}}\right) Z_{1}\left(k_{1}\right) P_{\mathrm{L}}\left(k_{1}\right) Z_{1}\left(k_{2}\right) P_{\mathrm{L}}\left(k_{2}\right) Z_{1}\left(k_{3}\right)\right) P_{\mathrm{L}}\left(k_{3}\right)+(3 \text { perms. })\right]
\end{aligned}
$$

where $Z_{n}$ is the $n$th order Eulerian perturbation kernel of the matter density field in the redshift space $[68,69]$. The tree-level covariance of the multipole power spectra is written as $[39,70]$

$$
\begin{aligned}
\operatorname{Cov}_{\ell \ell^{\prime}}^{(\text {tree })}\left(k_{i}, k_{j}\right)= & \frac{1}{V} \int_{\hat{\mathbf{k}}_{i \ell}} \int_{\hat{\mathbf{k}}_{j \ell^{\prime}}^{\prime}} T^{(\text {tree })}\left(\mathbf{k},-\mathbf{k}, \mathbf{k}^{\prime},-\mathbf{k}^{\prime}\right) \\
= & \frac{1}{V} \int_{\hat{\mathbf{k}}_{i \ell}} \int_{\hat{\mathbf{k}}_{j \ell^{\prime}}^{\prime}}\left[12 Z_{3}\left(\mathbf{k},-\mathbf{k}, \mathbf{k}^{\prime}\right) Z_{1}(k)^{2} Z_{1}\left(k^{\prime}\right) P_{L}(k)^{2} P_{L}\left(k^{\prime}\right)+8 Z_{2}\left(\mathbf{k}-\mathbf{k}^{\prime}, \mathbf{k}^{\prime}\right)^{2} Z_{1}\left(k^{\prime}\right)^{2} P_{L}\left(k^{\prime}\right)^{2} P_{L}\left(\left|\mathbf{k}^{\prime}-\mathbf{k}\right|\right)\right. \\
& \left.+8 Z_{2}\left(\mathbf{k}-\mathbf{k}^{\prime}, \mathbf{k}^{\prime}\right) Z_{2}\left(\mathbf{k}^{\prime}-\mathbf{k}, \mathbf{k}\right) P_{L}\left(\left|\mathbf{k}^{\prime}-\mathbf{k}\right|\right) Z_{1}(k) P_{L}(k) Z_{1}\left(k^{\prime}\right) P_{L}\left(k^{\prime}\right)+\left(\mathbf{k} \leftrightarrow \mathbf{k}^{\prime}\right)\right],
\end{aligned}
$$

where the integral denotes

$$
\int_{\hat{\mathbf{k}}_{i \ell}}=\int_{\mathbf{k} \in k_{i}} \frac{\mathbf{d k}}{V_{k_{i}}}(2 \ell+1) \mathcal{L}_{\ell}\left(\mu_{\mathbf{k}}\right)
$$

The tree-level covariance after reconstruction can be obtained by replacing $Z_{n}$ with the kernel of postreconstructed (postrec) spectra $Z_{n}^{(\text {rec })}$ derived by [58]. The first-order kernel is not changed by the reconstruction

$$
Z_{1}^{(\mathrm{rec})}(\mathbf{k})=Z_{1}(\mathbf{k})
$$

The relation of the second- and third-order postrec kernels to the prereconstructed (prerec) kernels is given as [58]

$$
Z_{2}^{(\mathrm{rec})}\left(\mathbf{k}_{1}, \mathbf{k}_{2}\right)=Z_{2}\left(\mathbf{k}_{1}, \mathbf{k}_{2}\right)+\frac{1}{2}\left[\left(\mathbf{k} \cdot \mathbf{S}^{\mathrm{z}(1)}\left(\mathbf{k}_{1}\right)\right)\left(\mathbf{k}_{2} \cdot \mathbf{L}^{z(1)}\left(\mathbf{k}_{2}\right)\right)+\left(\mathbf{k} \cdot \mathbf{S}^{\mathrm{z}(1)}\left(\mathbf{k}_{2}\right)\right)\left(\mathbf{k}_{1} \cdot \mathbf{L}^{z(1)}\left(\mathbf{k}_{1}\right)\right)\right]
$$

and

$$
\begin{aligned}
Z_{3}^{(\mathrm{rec})}\left(\mathbf{k}_{1}, \mathbf{k}_{2}, \mathbf{k}_{3}\right)= & Z_{3}\left(\mathbf{k}_{1}, \mathbf{k}_{2}, \mathbf{k}_{3}\right)+\frac{1}{6}\left[2\left(\mathbf{k} \cdot \mathbf{S}^{z(1)}\left(\mathbf{k}_{1}\right)\right) Z_{2}\left(\mathbf{k}_{2}, \mathbf{k}_{3}\right)+\left(\mathbf{k} \cdot \mathbf{S}^{z(1)}\left(\mathbf{k}_{1}\right)\right)\left(\mathbf{k} \cdot \mathbf{S}^{z(1)}\left(\mathbf{k}_{2}\right)\right)\left(\mathbf{k}_{\mathbf{3}} \cdot \mathbf{L}^{z(1)}\left(\mathbf{k}_{3}\right)\right)\right. \\
& \left.+\left(\mathbf{k} \cdot \mathbf{S}^{z(2)}\left(\mathbf{k}_{1}, \mathbf{k}_{2}\right)\right)\left(\mathbf{k}_{\mathbf{3}} \cdot \mathbf{L}^{z(1)}\left(\mathbf{k}_{\mathbf{3}}\right)\right)+(2 \text { perms. })\right],
\end{aligned}
$$

where $\mathbf{k}=\mathbf{k}_{1}+\cdots+\mathbf{k}_{n}$ in the $n$th order kernel. In the above equations, $\mathbf{L}^{z(n)}$ represents the $n$th order Lagrangian kernel in redshift space, which is related to the same order of Lagrangian kernel in real space $\mathbf{L}^{(n)}$ as [69]

$$
\mathbf{L}^{z(n)}=\mathbf{R}^{(n)} \mathbf{L}^{(n)} .
$$

The redshift-space distortion tensor at $n$th order $\mathbf{R}^{(n)}$ is given by 

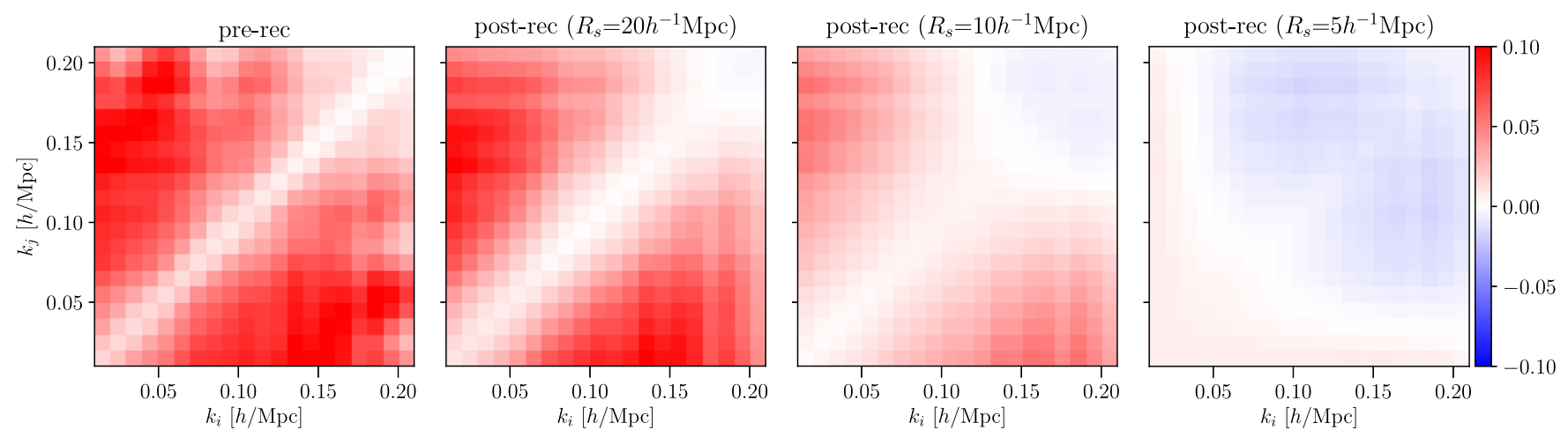

FIG. 1. Tree-level non-Gaussian covariance of the monopole component of the matter power spectrum normalized with their Gaussian covariance, i.e., $\operatorname{Cov}_{00}^{(\text {tree })}\left(k_{i}, k_{j}\right) /\left[\operatorname{Cov}_{00}^{(\mathrm{G})}\left(k_{i}, k_{i}\right) \mathbf{C o v}_{00}^{(\mathrm{G})}\left(k_{j}, k_{j}\right)\right]^{1 / 2}$ for prerec and postrec spectra with $R_{s}=20 h^{-1} \mathrm{Mpc}, 10 h^{-1} \mathrm{Mpc}$, and $5 h^{-1} \mathrm{Mpc}$ from left to right panels. As $R_{s}$ is smaller, the off-diagonal terms are changed from positive to negative values. The output redshift is $z=1.02$.

$$
\mathbf{R}_{i j}^{(n)}=\delta_{i j}+n f \hat{z}_{i} \hat{z}_{j}
$$

where $\delta_{i j}$ is the Kronecker delta and $\hat{z}_{i}$ the ith component of the unit vector in the line-of-sight direction. In a standard reconstruction [42], the shift field is estimated from the smoothed density field using the inverse Zeldovich approximation. The $n$th order kernel of the shift field $\mathbf{S}^{z(n)}$ is then given by

$\mathbf{S}^{z(n)}\left(\mathbf{k}_{1}, \ldots, \mathbf{k}_{n}\right)=-n ! W(k) \mathbf{L}^{(1)}(\mathbf{k}) Z_{n}\left(\mathbf{k}_{1}, \ldots, \mathbf{k}_{n}\right)$,

where $W(k)$ is the smoothing kernel and we adopt Gaussian kernel, i.e., $W(k)=\exp \left(-k^{2} R_{s}^{2} / 2\right)$ with different smoothing scales $R_{s}=5 h^{-1} \mathrm{Mpc}, 10 h^{-1} \mathrm{Mpc}$, and $20 h^{-1} \mathrm{Mpc}$.

Figure 1 compares the tree-level non-Gaussian covariance of the monopole spectra before and after the reconstruction. The plotted covariance is normalized with their Gaussian components, i.e., $\operatorname{Cov}_{00}^{(\text {tree })}\left(k_{i}, k_{j}\right) /$ $\left[\operatorname{Cov}_{00}^{(\mathrm{G})}\left(k_{i}, k_{i}\right) \operatorname{Cov}_{00}^{(\mathrm{G})}\left(k_{j}, k_{j}\right)\right]^{1 / 2}$. The off-diagonal components have positive values for the prerec spectra, which means that the different modes are positively correlated by the mode coupling of gravity. After reconstruction, we find that the positive correlation decreases and becomes negative at $R_{s}$ less than $10 h^{-1} \mathrm{Mpc}$. This comes from that the values of the tree-level trispectra shift from positive to negative by replacing the perturbative kernels with the reconstructed one. This is related to our previous finding that the amplitudes of the one-loop terms of the power spectrum given by the same perturbative kernels decrease after the reconstruction [58].

\section{III. $N$-BODY SIMULATIONS}

We measure the covariance of the multipole components of matter power spectra over an ensemble of dark matter $N$-body simulations as follows:

$$
\begin{aligned}
& \operatorname{Cov}_{\ell \ell^{\prime}}\left(k, k^{\prime}\right) \\
& \quad=\frac{1}{N_{\text {real }}-1} \sum_{i}^{N_{\text {real }}}\left[P_{\ell, i}(k)-\bar{P}_{\ell}(k)\right]\left[P_{\ell^{\prime}, i}\left(k^{\prime}\right)-\bar{P}_{\ell^{\prime}}\left(k^{\prime}\right)\right],
\end{aligned}
$$

where $N_{\text {real }}$ is the number of realizations and $\bar{P}_{\ell}$ is the averaged multipole components of matter power spectra. The binning width of $k$ is uniformly set to be $0.01 h / \mathrm{Mpc}$ and the minimum value of $k$ is set to be $0.01 \mathrm{~h} / \mathrm{Mpc}$. We perform $N$-body simulations using a publicly available code GADGET-2 [71]. The initial distribution of mass particles is based on the 2LPT code [72,73] with Gaussian initial conditions at the input redshift of 31 . The initial redshift does not affect (less than 1\%) the power spectrum at $k<0.3 h / \mathrm{Mpc}$ and $z=0$ (see Fig. 35 of [74]). The initial linear power spectrum is computed by CAMB [75]. We adopt 4000 realizations with $512^{3}$ mass particles in a cubic box with a side length of $500 h^{-1} \mathrm{Mpc}$ and two output redshifts of $z=0$ and $z=1.02$.

The $N$-body particles are assigned to $512^{3}$ grid cells with the clouds-in-cell (CIC) method to calculate the density contrast. We then perform the Fourier transform [76] to measure the multipole components of the power spectra $P_{\ell}$ after the pixel window effect is corrected [e.g., 77]. The reconstructed density field is computed as follows [42]:

(i) The shift field for the reconstruction is computed from the smoothed redshift-space mass density field using the inverse Zeldovich approximation, i.e., $\tilde{\mathbf{s}}(\mathbf{k})=$ $-\left(\mathbf{k} / k^{2}\right) \tilde{\delta}_{m}^{(\mathrm{z})}(\mathbf{k}) W(k)$ with Gaussian smoothing kernel $W(k)=\exp \left(-k^{2} R_{s}^{2} / 2\right)$ at $R_{s}=5 h^{-1} \mathrm{Mpc}$, $10 h^{-1} \mathrm{Mpc}$, and $20 h^{-1} \mathrm{Mpc}$. Note that we leave the reconstructed field anisotropic on large scales to constrain the growth rate from the anisotropy due to the redshift-space distortion. 
(ii) Each mass particle is displaced following the above shift field at the position interpolated from the shift field at neighboring grids with the CIC scheme.

(iii) Random particles are also displaced using the same shift vector field in the same manner as the mass particles.

(iv) Reconstructed density field is obtained by the displaced random field subtracted from the displaced data field as $\delta^{(\mathrm{rec})}=\delta_{\mathrm{d}}^{(\mathrm{rec})}-\delta_{\mathrm{r}}^{(\mathrm{rec})}$.

We also compute the matter power spectra from eight realizations of large-box $N$-body simulations with a side length of $4 h^{-1} \mathrm{Gpc}$. Each realization contains $4096^{3}$ mass particles and they are assigned to $2048^{3}$ grid cells with the CIC method. We confirm that the averaged spectra from
$500 h^{-1} \mathrm{Mpc}$ box is consistent with those from $4 h^{-1} \mathrm{Gpc}$ box; however, we find a large fluctuation of the quadrupole spectrum from $500 \mathrm{~h}^{-1} \mathrm{Mpc}$ box due to a low resolution in $k$-space. We therefore adopt the power spectrum from $4 h^{-1}$ Gpc-box simulations and add the following correction to the covariance from $500 \mathrm{~h}^{-1} \mathrm{Mpc}$ box simulations as

$$
\begin{aligned}
\operatorname{Cov}_{\ell \ell^{\prime}}\left(k, k^{\prime}\right)= & \operatorname{Cov}_{\ell \ell^{\prime}}^{\left(500 \mathrm{~h}^{-1} \mathrm{Mpc}\right)}\left(k, k^{\prime}\right) \\
& \times\left[\frac{\bar{P}_{\ell}^{\left(4 \mathrm{~h}^{-1} \mathrm{Gpc}\right)}(k)}{\bar{P}_{\ell}^{\left(500 \mathrm{~h}^{-1} \mathrm{Mpc}\right)}(k)}\right]\left[\frac{\bar{P}_{\ell^{\prime}}^{\left(4 \mathrm{~h}^{-1} \mathrm{Gpc}\right)}\left(k^{\prime}\right)}{\bar{P}_{\ell^{\prime}}^{\left(500 \mathrm{~h}^{-1} \mathrm{Mpc}\right)}\left(k^{\prime}\right)}\right],
\end{aligned}
$$
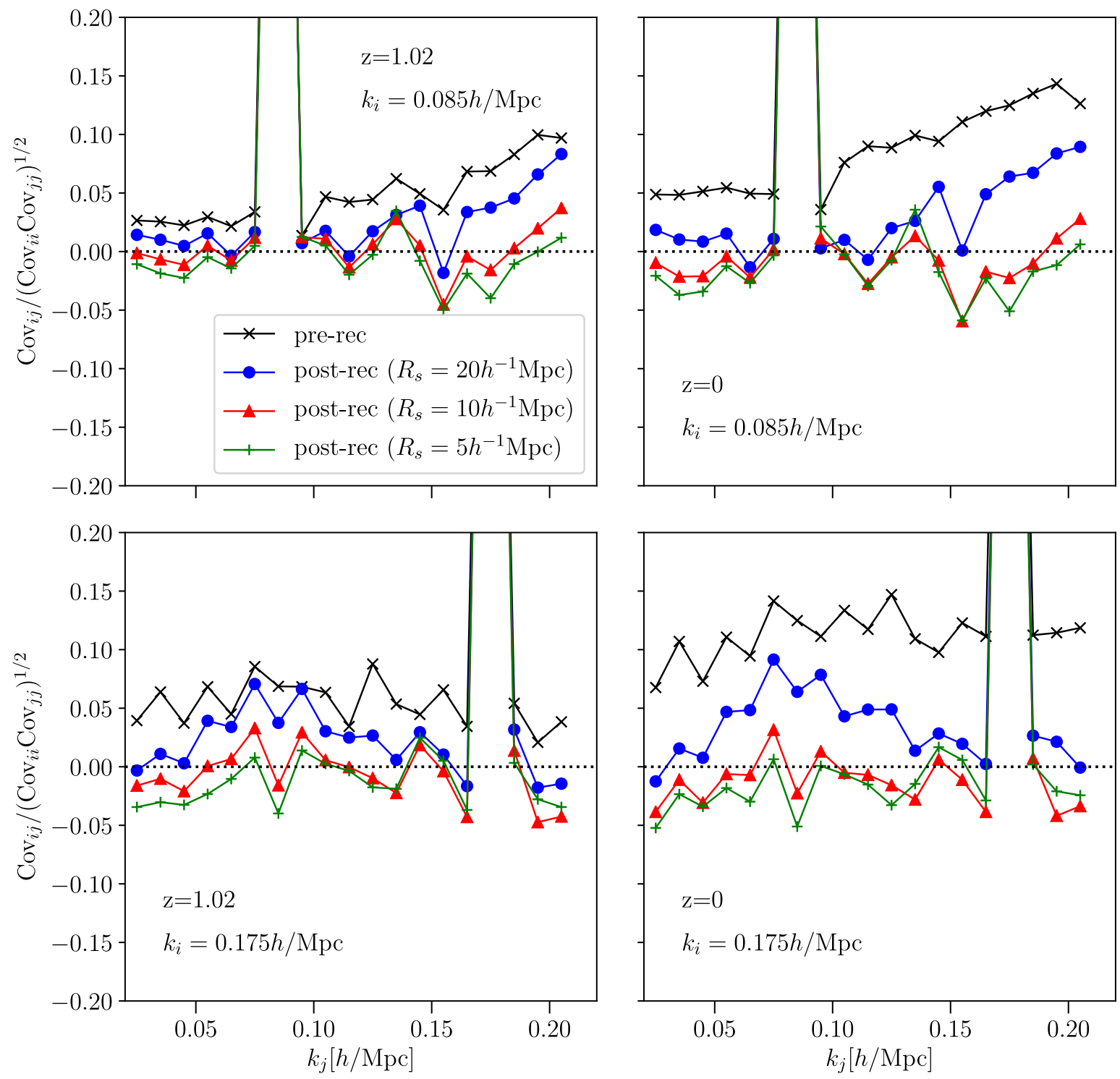

FIG. 2. Correlation coefficients of the monopole components of the matter power spectrum with fixed $k_{i}=0.085 h / \mathrm{Mpc}$ (upper) and $0.175 \mathrm{~h} / \mathrm{Mpc}$ (lower) at $z=1.02$ (left) and $z=0$ (right). Different symbols denote the results for prerec and postrec spectra with different $R_{s}$. Off-diagonal components of postrec spectra significantly decrease and have negative values at $R_{s}$ less than $10 h^{-1} \mathrm{Mpc}$, which is consistent with the behavior of the perturbation theory in Fig. 1. 
where $\bar{P}_{\ell}^{\left(L_{\text {box }}\right)}$ is the averaged multipole power spectrum from $N$-body simulations with a side length of $L_{\text {box }}$.

Figure 2 shows the correlation matrix of the monopole spectra computed from the simulations at fixed $k_{i}=$ $0.085 h / \mathrm{Mpc}$ and $0.175 h / \mathrm{Mpc}$ at $z=1.02$ and 0 . Prerec spectra are positively correlated among different modes and thus the off-diagonal components are positive [3,39]. We find that the off-diagonal components substantially decrease to be nearly zero by reconstruction with $R_{s}=10 h^{-1} \mathrm{Mpc}$. At $R_{s}=5 h^{-1} \mathrm{Mpc}$, the off-diagonal components become negative values. This behavior is qualitatively consistent with the perturbation theory shown in the previous section.

\section{SIGNAL-TO-NOISE RATIO OF MULTIPOLE POWER SPECTRA}

In this section, we evaluate the information content of redshift-space matter power spectra from the following signal-to-noise ratio $(\mathrm{S} / \mathrm{N})$ :

$(\mathrm{S} / \mathrm{N})^{2}=\sum_{\ell, \ell^{\prime}}^{0,2} \sum_{i, j}^{k_{i}, k_{j} \leq k_{\max }} P_{\ell}\left(k_{i}\right)\left(\mathbf{C o v}^{-1}\right)_{\ell \ell^{\prime}}\left(k_{i}, k_{j}\right) P_{\ell^{\prime}}\left(k_{j}\right)$.

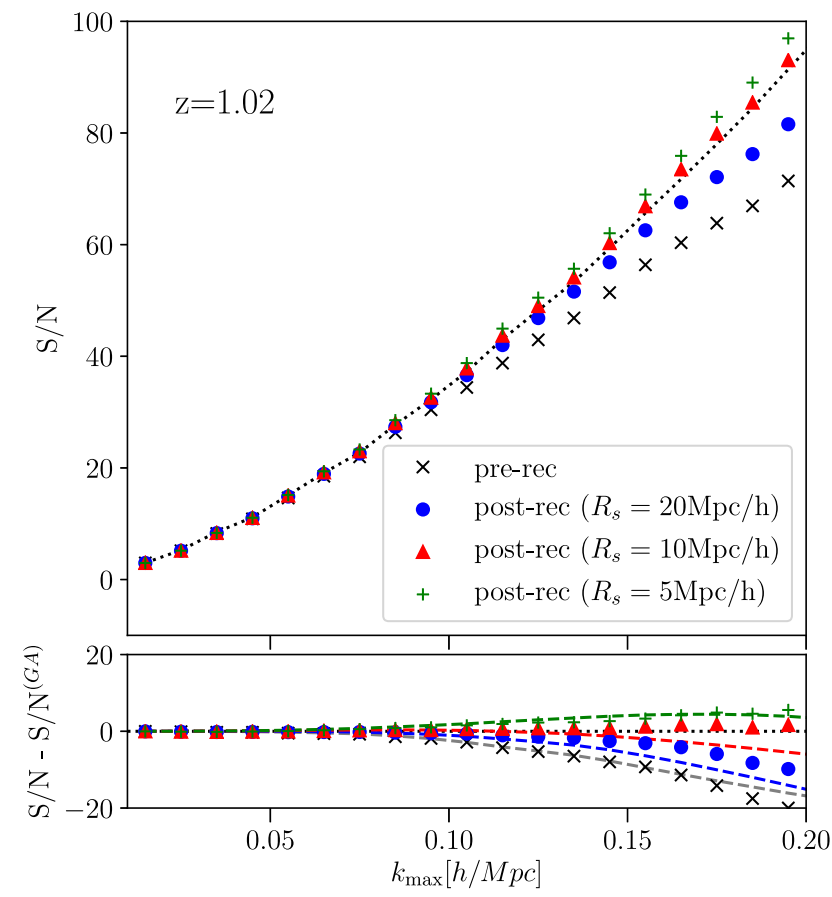

The multipole spectra $P_{\ell}$ and their covariance are directly estimated from the simulations as explained in the previous section. The inverse covariance matrix is computed by multiplying a so-called Hartlap factor $\alpha=\left(N_{\text {real }}-N_{\text {bin }}-2\right) /$ $\left(N_{\text {real }}-1\right)$ with the inverse of the covariance matrix [Eq. (14)] [78].

Figure 3 compares the $\mathrm{S} / \mathrm{N}$ of prerec spectra and postrec spectra with different $R_{S}$ as a function of the maximum wave number $k_{\max }$. We find that the postrec spectra have higher $\mathrm{S} / \mathrm{N}$ than the prerec spectra. The improvement is larger at higher $k$. For example, the $\mathrm{S} / \mathrm{N}$ of the postrec spectra with $R_{s}=$ $10 \mathrm{~h}^{-1} \mathrm{Mpc}$ is improved by $7 \%\left(k_{\max }=0.1 \mathrm{~h} / \mathrm{Mpc}\right)$ and $30 \%\left(k_{\max }=0.2 h / \mathrm{Mpc}\right)$ at $z=1.02$ relative to the prerec spectra. The improvement is more significant at $z=0: 18 \%$ $\left(k_{\max }=0.1 \mathrm{~h} / \mathrm{Mpc}\right)$ and $69 \%\left(k_{\max }=0.2 \mathrm{~h} / \mathrm{Mpc}\right)$. Since the diagonal components of the covariance matrix are dominated by the Gaussian terms, the improvement of the $\mathrm{S} / \mathrm{N}$ mainly comes from the decrement of the off-diagonal components as shown in Figs. 1 and 2.

We also plot the $\mathrm{S} / \mathrm{N}$ estimated from the linear spectra and the Gaussian covariance as a reference. Lower panels focus on the differences of $\mathrm{S} / \mathrm{N}$ from the linear Gaussian one. Interestingly, it is found that the $\mathrm{S} / \mathrm{N}$ of the postrec spectra at $R_{s}=10 h^{-1} \mathrm{Mpc}$ and $5 h^{-1} \mathrm{Mpc}$ is comparable to or higher than the linear Gaussian one. The similar trend

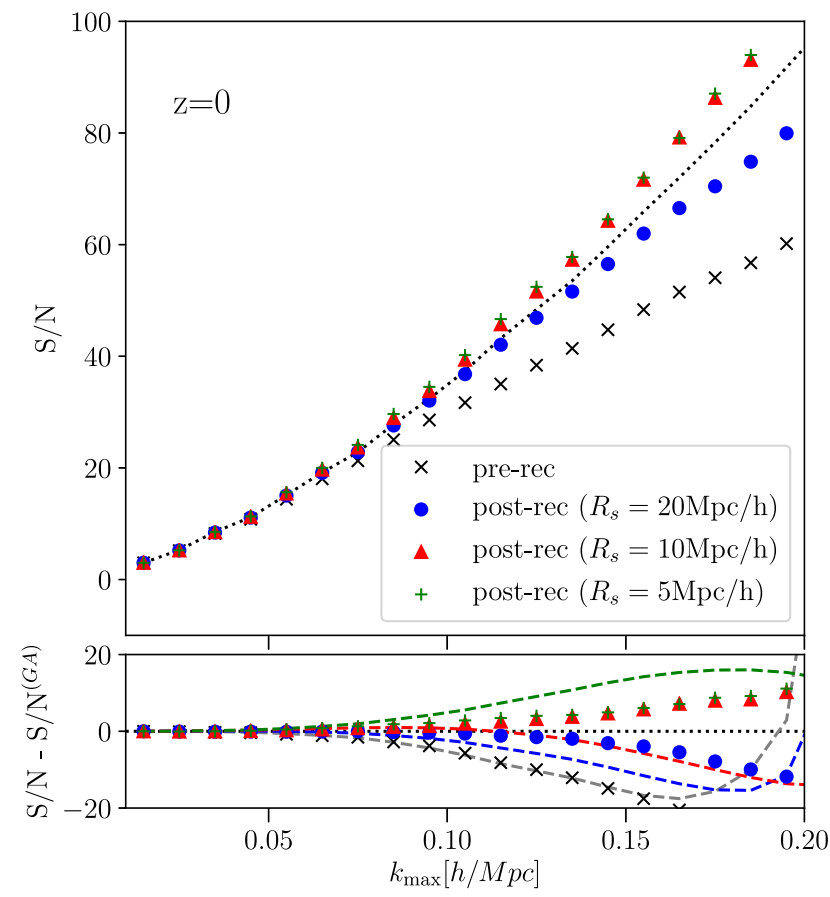

FIG. 3. Comparison of the $\mathrm{S} / \mathrm{N}$ of the sum of the monopole and quadrupole components of redshift-space matter power spectra before reconstruction and after reconstruction with $R_{s}=20 h^{-1} \mathrm{Mpc}, 10 h^{-1} \mathrm{Mpc}$, and $5 h^{-1} \mathrm{Mpc}$ at $z=1.02$ (left) and $z=0$ (right). For comparison, we plot the $\mathrm{S} / \mathrm{N}$ of the linear power spectrum using the analytical Gaussian covariance $\left(\mathrm{Cov}^{(\mathrm{GA})}\right)$ with dotted lines. We find that the postrec spectra have a better $\mathrm{S} / \mathrm{N}$ than prerec one and also that the postrec spectra with $R_{s}=10 h^{-1} \mathrm{Mpc}$ and $5 h^{-1} \mathrm{Mpc}$ have higher $\mathrm{S} / \mathrm{N}$ than the Gaussian one. Lower panels focus on the differences of the $\mathrm{S} / \mathrm{N}$ for the linear Gaussian one. For comparison, the $\mathrm{S} / \mathrm{N}$ of the linear power spectra using the tree-level perturbative covariance $\left(\mathrm{Cov}^{(\mathrm{GA})}+\mathrm{Cov}^{(\text {tree })}\right)$ is plotted with dashed lines and shows the similar behavior. 

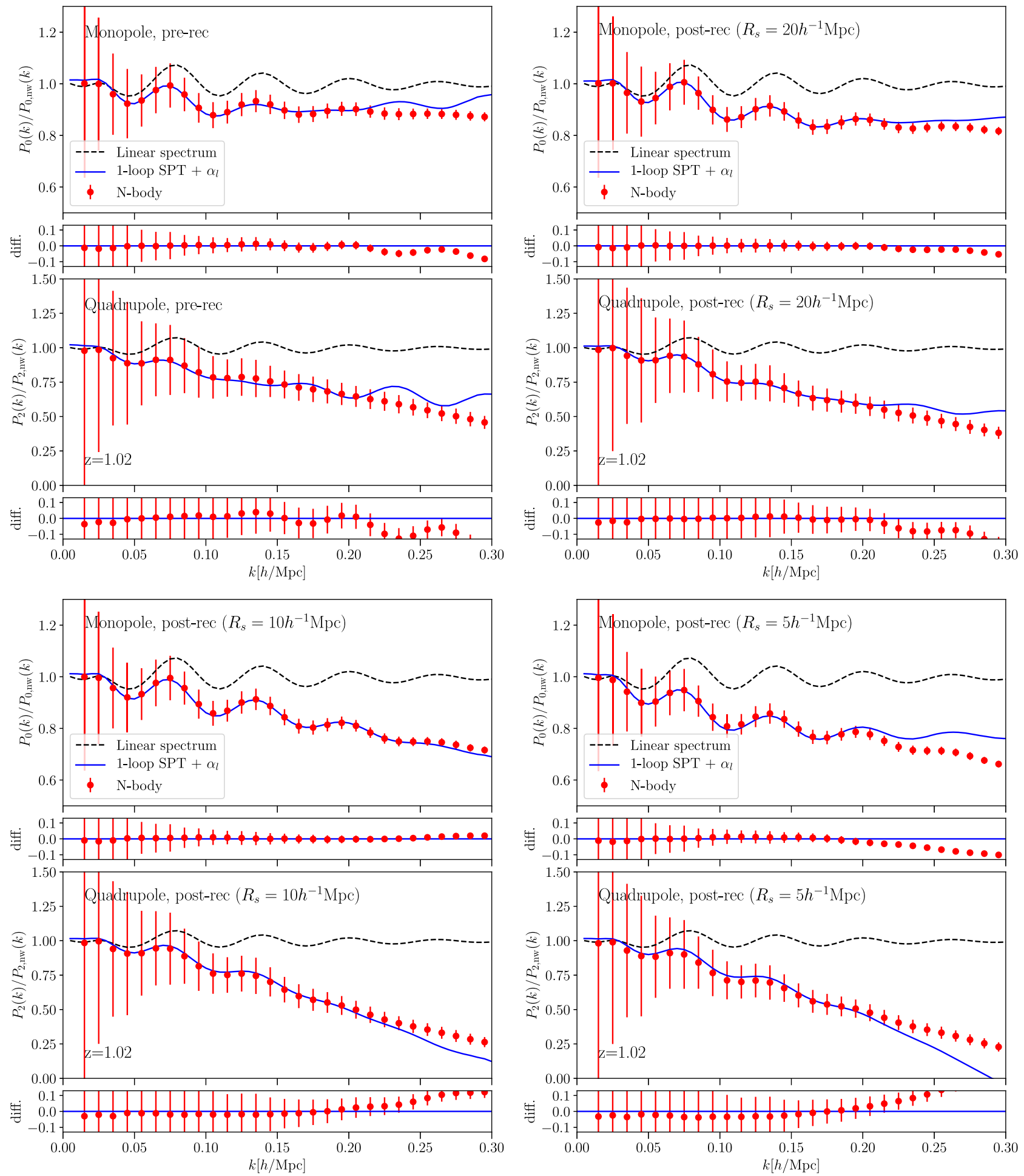

FIG. 4. Monopole and quadruple components of the matter power spectrum from $N$-body simulations (filled circles) before reconstruction (upper-left) and after reconstruction with $R_{s}=20 h^{-1} \mathrm{Mpc}$ (upper-right), $10 h^{-1} \mathrm{Mpc}$ (lower-left), and $5 h^{-1} \mathrm{Mpc}$ (lower-right). Error bars denote the $1 \sigma$ sample variance from $4000 \mathrm{~N}$-body simulations where each volume is $\left(500 \mathrm{~h}^{-1} \mathrm{Mpc}\right)^{3}$. For comparison, the one-loop perturbative formula is plotted with solid lines using the best-fit values of the lowest-order counterterms $\alpha_{\ell}$ $(\ell=0,2)$ up to $k_{\max }=0.2 \mathrm{~h} / \mathrm{Mpc}$. The linear power spectra are also plotted with dashed lines. All of the plotted spectra are divided with the no-wiggle spectra. Small panels show the differences between the simulated spectra and the one-loop perturbative formula with the best-fit values of $\alpha_{\ell}$. The output redshift is $z=1.02$. 

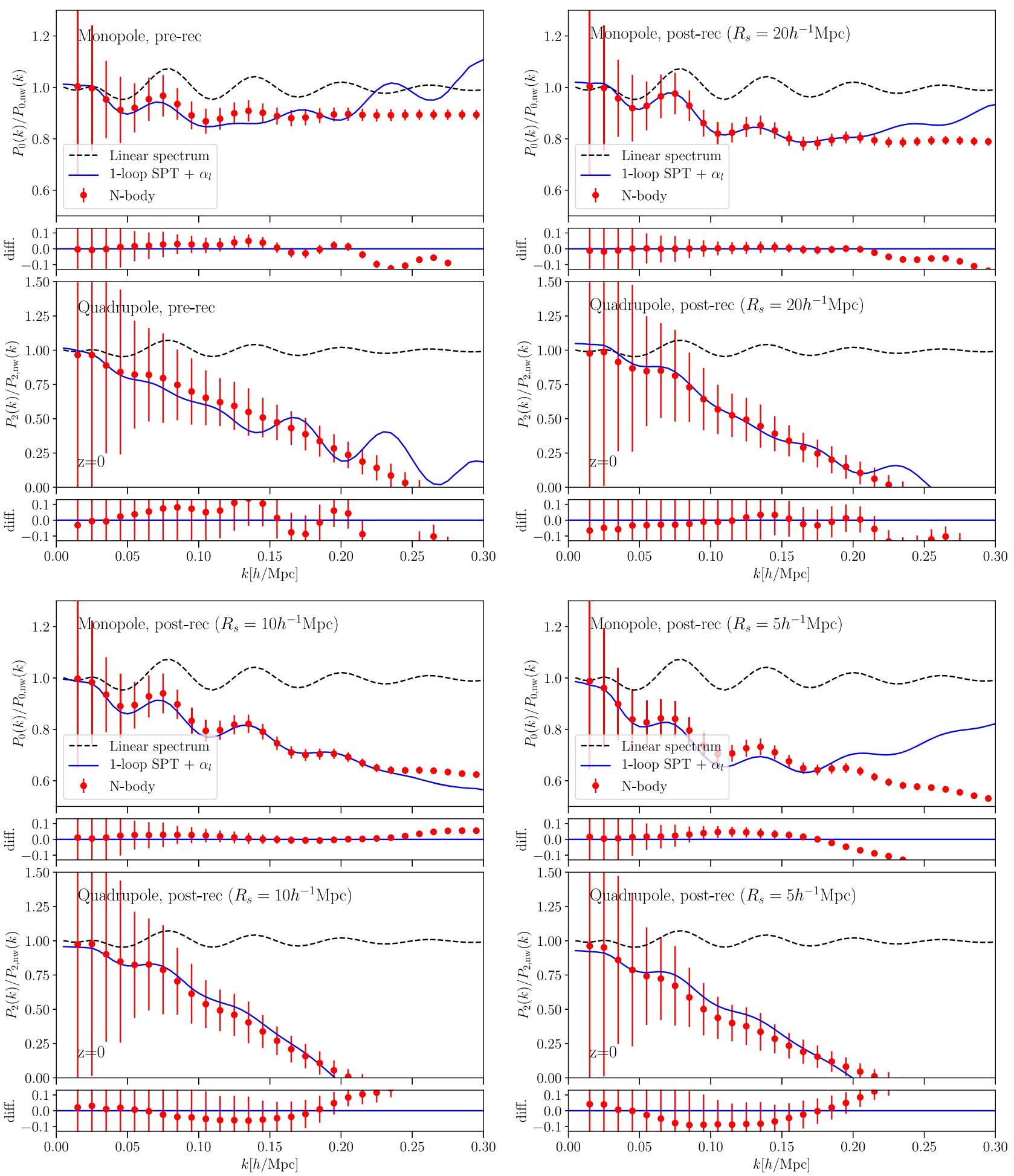

FIG. 5. Same as Fig. 4 but for the output redshift of $z=0$.

is found from the perturbative predictions where the linear spectra and the tree-level covariance [Eq. (6)] are applied to calculate the S/N [Eq. (16)], though the agreement of the perturbation with the numerical results is limited to be at $k \leq 0.1 h / \mathrm{Mpc}$. The $\mathrm{S} / \mathrm{N}$ from the perturbation rapidly increases at high $k$ because the determinant of tree-level covariance diverges [40]. As shown in Figs. 1 and 2, the off-diagonal components become negative at $R_{s} \lesssim$ $\sim 10 h^{-1} \mathrm{Mpc}$ and thereby the $\mathrm{S} / \mathrm{N}$ of the postrec spectra becomes higher than the linear Gaussian one.

Information of nonlinear growth of structure can be normally captured by higher-order statistics beyond 
two-point statistics. The reconstruction returns the information leaking to higher-order statistics back to the twopoint statistics. The return is larger at smaller $R_{s}$ where smaller scales can be reconstructed and thereby the $\mathrm{S} / \mathrm{N}$ increases at smaller $R_{s}$. The growth information is however buried on strongly nonlinear regime, and thus the increment of $\mathrm{S} / \mathrm{N}$ from $R_{s}=10 h^{-1} \mathrm{Mpc}$ to $R_{s}=5 h^{-1} \mathrm{Mpc}$ at $z=0$ is limited.

\section{IMPACTS ON GROWTH RATE MEASUREMENTS}

In this section, we explore if the estimates of cosmological parameters are improved by reconstruction, particularly focusing on the growth rate. We evaluate the error of the growth rate including the systematics when using the one-loop perturbative formulas as a theoretical modeling of the matter power spectra. The likelihood is estimated as follows:

$$
\mathcal{L} \propto \exp \left(-\frac{\chi^{2}}{2}\right)
$$

$$
\begin{aligned}
\chi^{2}(\mathbf{p})= & \sum_{\ell, \ell^{\prime}}^{0,2} \sum_{i, j}^{k_{i}, k_{j} \leq k_{\max }}\left[P_{\ell}^{\text {theory }}\left(k_{i} ; \mathbf{p}\right)-P_{\ell}^{\operatorname{sim}}\left(k_{i}\right)\right] \\
& \times\left(\mathbf{C o v}^{-1}\right)_{\ell \ell^{\prime}}\left(k_{i}, k_{j}\right)\left[P_{\ell^{\prime}}^{\text {theory }}\left(k_{j} ; \mathbf{p}\right)-P_{\ell^{\prime}}^{\operatorname{sim}}\left(k_{j}\right)\right] .
\end{aligned}
$$

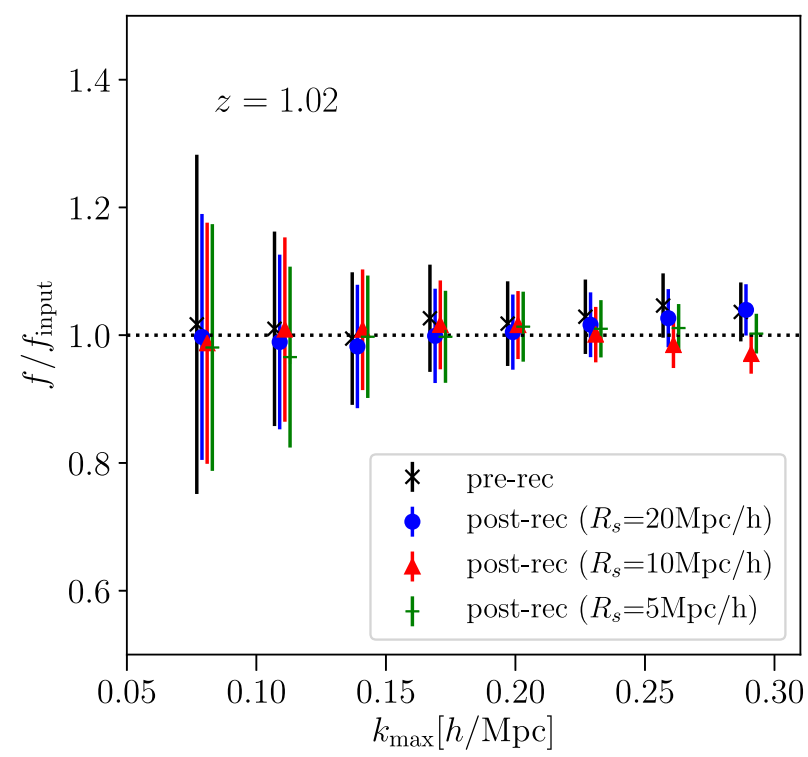

For the theoretical model, we adopt the one-loop perturbative formulas derived in our previous work [58]. In order to correct the effects from small-scale physics on large-scale modes, we include the lowest-order counterterms given by $\alpha_{\ell}(\ell=0,2)$ multiplied with $k^{2}$ times the linear power spectrum $P_{\ell}^{\text {linear }}$ as suggested from the effective field theory [e.g., 79],

$$
P_{\ell}^{\text {theory }}=P_{\ell}^{1-\text { loop }}+\alpha_{\ell} k^{2} P_{\ell}^{\text {linear }}(k) .
$$

The set of free parameters $\mathbf{p}$ is the growth rate $f$ and two counterterms $\alpha_{\ell}$ and other cosmological parameters are fixed for simplicity. In the theoretical covariance, $P_{\ell}$ is again estimated from simulations with the survey volume $V=\left(500 h^{-1} \mathrm{Mpc}\right)^{3}$ and the number density $n \sim$ $1\left[\left(h^{-1} \mathrm{Mpc}\right)^{-3}\right]$ including a volume-size correction [Eq. (15)], which fully takes into account the mode coupling between different bins of $k$. Note that in our previous paper [58] we assumed the Gaussian covariance with different volumes and number density for simplicity to estimate the impact of the growth rate measurement. We estimate the posterior distribution using a nested sampling algorithm MULTINEST [80], implemented in MONTE PYTHON [81].

Figures 4 and 5 compare the monopole and quadrupole spectra from $N$-body simulations with the one-loop perturbation theory at $z=1.02$ and 0 . We adopt the best-fit values of $\alpha_{\ell}$ by fitting the spectrum out to $k=0.2 h / \mathrm{Mpc}$. Each panel shows the results of prerec (upper-left) and postrec spectra with different $R_{s}=20 h^{-1} \mathrm{Mpc}$ (upper-right),

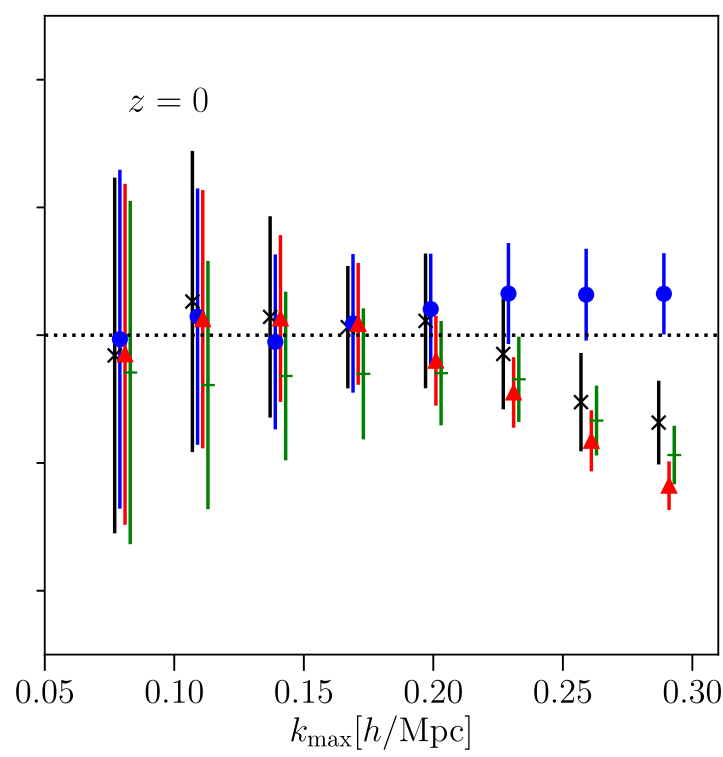

FIG. 6. Linear growth rate relative to the input value obtained by fitting the one-loop perturbative formulae of the monopole and quadrupole power spectra to the simulated spectra with the maximum wave number $k_{\max }$ varied [Eq. (17)]. The counterterms $\alpha_{\ell}(\ell=0$ and 2) are freely fitted, while other cosmological parameters are fixed. Different plots show the prerec spectra (x-shaped crosses) and postrec spectra with $R_{s}=20 h^{-1} \mathrm{Mpc}$ (circles), $10 \mathrm{~h}^{-1} \mathrm{Mpc}$ (triangles), and $5 \mathrm{~h}^{-1} \mathrm{Mpc}$ (+-shaped crosses) at $z=1.02$ (left panels) and $z=0$ (right panels). Covariance of the multipole power spectra is estimated from 4000 realizations of $N$-body results with $\left(500 h^{-1} \mathrm{Mpc}\right)^{3}$ volume. The error bars denote the $1 \sigma$ uncertainty. 
$10 h^{-1} \mathrm{Mpc}$ (lower-left), and $5 h^{-1} \mathrm{Mpc}$ (lower-right). We find that the fitting to the simulated spectrum is best for the postrec spectra with $R_{s}=20 h^{-1} \mathrm{Mpc}$, while the postrec spectra with $R_{s}=5 h^{-1} \mathrm{Mpc}$ is the worst fitting. More quantitatively speaking, the minimum chi-squared values are 0.76 (prerec), 0.09 (postrec with $20 h^{-1} \mathrm{Mpc}$ ), 0.88 (postrec with $10 h^{-1} \mathrm{Mpc}$ ), and 3.6 (postrec with $5 h^{-1} \mathrm{Mpc}$ ) at $z=1.02$ and 8.9 (prerec), 0.7 (postrec with $20 h^{-1} \mathrm{Mpc}$ ), 4.7 (postrec with $10 h^{-1} \mathrm{Mpc}$ ), and 27 (postrec with $5 h^{-1} \mathrm{Mpc}$ ). The authors in Ref. [58] have described that the reconstruction partially suppresses the nonlinearity of the gravitational growth and thereby the perturbation works at higher $k$. However, when $R_{s}$ is too small, the shift field estimated from the evolved density field becomes more nonlinear and thereby the perturbation does not work well. This is consistent with our previous work in real-space matter clustering [57].

Figure 6 shows the best-fit values of $f$ against the input value $f_{\text {input }}$ and its 1 sigma error. We find that the value of $f / f_{\text {input }}$ is consistent with unity up to $k_{\max } \sim 0.3 \mathrm{~h} / \mathrm{Mpc}$ at $z=1.02$ and $k_{\max } \sim 0.2 \mathrm{~h} / \mathrm{Mpc}$ at $z=0$ for both prerec and postrec spectra. Note that since the one-loop approximation does not work at such high $k_{\max }$, the input value of $f$ can be recovered by chance. It is found that the statistical error decreases after reconstruction over all range of $k$. For example, the error decrements of $f$ from the postrec spectra relative to that from the prerec spectra at $z=1.02$ are $11 \%$ $\left(R_{s}=20 h^{-1} \mathrm{Mpc}\right), 20 \%\left(R_{s}=10 h^{-1} \mathrm{Mpc}\right)$, and $17 \%$ $\left(R_{s}=5 h^{-1} \mathrm{Mpc}\right) \quad$ at $\quad k_{\max }=0.2 h^{-1} \mathrm{Mpc}$ and $13 \%$ $\left(R_{s}=20 h^{-1} \mathrm{Mpc}\right), 33 \%\left(R_{s}=10 h^{-1} \mathrm{Mpc}\right)$, and $33 \%$ $\left(R_{s}=5 h^{-1} \mathrm{Mpc}\right)$ at $k_{\max }=0.3 h^{-1} \mathrm{Mpc}$. The error decrements at $z=0$ are $18 \%\left(R_{s}=20 h^{-1} \mathrm{Mpc}\right), 33 \%$ $\left(R_{s}=10 h^{-1} \mathrm{Mpc}\right)$, and $22 \%\left(R_{s}=5 h^{-1} \mathrm{Mpc}\right)$ when $k_{\max }=0.2 h^{-1} \mathrm{Mpc}$. We find that the error of $f$ is significantly improved and the error improvement is almost maximized around $R_{s} \sim 10 \mathrm{~h}^{-1} \mathrm{Mpc}$ where the covariance is almost diagonal by reconstruction. Table I summarizes the minimum chi-squared values and the reduction of statistical errors for prerec and postrec spectra with three different smoothing scales.

\section{SUPERSAMPLE COVARIANCE}

The SSC comes from the mixing between the longwavelength modes beyond the survey window and the short-wavelength modes inside the survey area. The response of the power spectrum to the change in background density $\delta_{b}$ is given as $[64,65]$

$$
\operatorname{Cov}_{\ell \ell^{\prime}}^{(\mathrm{SSC})}=\sigma_{\mathrm{b}}^{2} \frac{\partial P_{\ell}(k)}{\partial \delta_{\mathrm{b}}} \frac{\partial P_{\ell^{\prime}}\left(k^{\prime}\right)}{\partial \delta_{\mathrm{b}}},
$$

where the variance of $\delta_{\mathrm{b}}$ in the survey window is defined as

$$
\sigma_{\mathrm{b}}^{2}=\frac{1}{V^{2}} \int \frac{\mathbf{d q}}{(2 \pi)^{3}}|\tilde{W}(\mathbf{q})|^{2} P_{L}(q),
$$

with the Fourier transform of the survey mask field $W(\mathbf{x})$ given as $\tilde{W}(\mathbf{q})$. The response of the multipole power spectrum to $\delta_{\mathrm{b}}$ is given by [82]

$$
\frac{\partial \ln P_{\ell}(k)}{\partial \delta_{\mathrm{b}}}=G_{\ell}+D_{\ell} \frac{d \ln k^{3} P_{\ell}(k)}{d \ln k},
$$

where the first term is the growth modulation by the background density, which is also known as beat coupling [63], and the second term is the dilation effect that comes from the change of the local expansion rate depending on the background density [65]. Here we neglect the response of the background tide for simplicity. The growth and dilation term for $\ell=0$ and 2 are given in Tables 1 and 2 of [82] as

$$
\begin{gathered}
G_{0}=\frac{\frac{68}{21}(1+f)+\frac{164}{105} f^{2}+\frac{4}{15} f^{3}}{1+\frac{2}{3} f+\frac{1}{5} f^{2}}, \\
G_{2}=\frac{\frac{122}{21} f+\frac{656}{147} f^{2}+\frac{58}{63} f^{3}}{\frac{4}{3} f+\frac{4}{7} f^{2}},
\end{gathered}
$$

\begin{tabular}{|c|c|c|c|c|c|}
\hline & & Prerec & $20 h^{-1} \mathrm{Mpc}$ & $10 h^{-1} \mathrm{Mpc}$ & $5 h^{-1} \mathrm{Mpc}$ \\
\hline \multirow{2}{*}{$\chi_{\min }^{2}$} & $z=1$ & 0.76 & 0.09 & 0.88 & 3.6 \\
\hline & $z=0$ & 8.9 & 0.7 & 4.7 & 27 \\
\hline Error & $z=1$ & & & & \\
\hline \multirow[t]{3}{*}{ Reduction } & $\begin{array}{c}0.2 h^{-1} \mathrm{Mpc} \\
z=1\end{array}$ & $\cdots$ & $11 \%$ & $20(13) \%$ & $17 \%$ \\
\hline & $\begin{array}{c}0.3 h^{-1} \mathrm{Mpc} \\
z=0\end{array}$ & $\ldots$ & $13 \%$ & $33(30) \%$ & $33 \%$ \\
\hline & $0.2 h^{-1} \mathrm{Mpc}$ & $\ldots$ & $18 \%$ & $33(28) \%$ & $22 \%$ \\
\hline
\end{tabular}

TABLE I. A summary of the minimum chi-squared values and the reduction of statistical errors for prerec and postrec spectra with three different smoothing scales $R_{s}$. For the reduction of statistical errors at $z=1$, the results with two different $k_{\max }$ are shown. The error reduction for $R_{s}=10 \mathrm{~h}^{-1} \mathrm{Mpc}$ shown in the bracket is the case with the supersample covariance. 

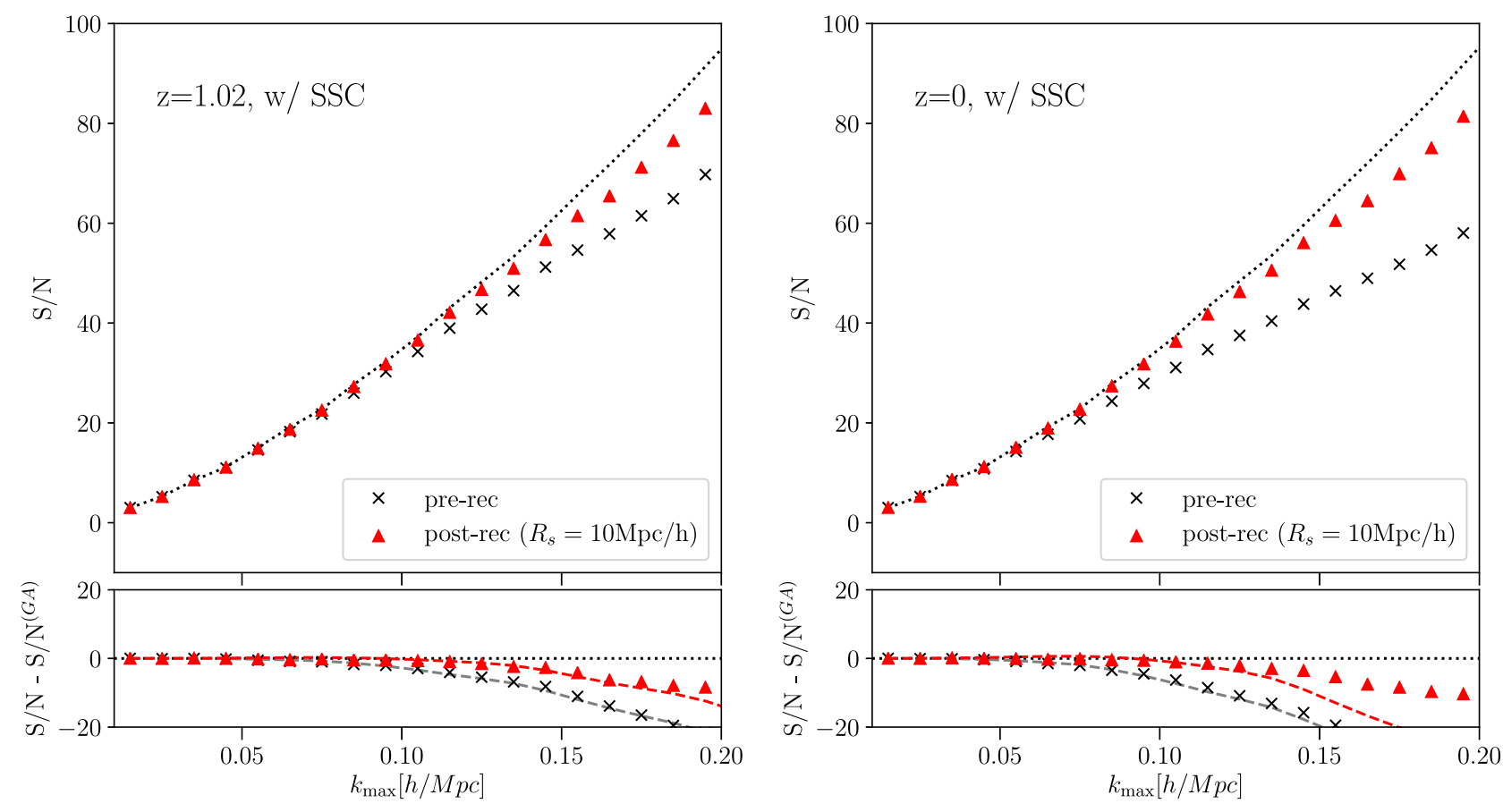

FIG. 7. Same as Fig. 3 but for the S/N of prerec and postrec spectra with $R_{s}=10 h^{-1} \mathrm{Mpc}$ and the SSC is included in the simulated covariance. The predictions from the one-loop perturbative formulas are also estimated from the covariance including SSC, i.e., $\mathrm{Cov}^{(\mathrm{GA})}+\mathrm{Cov}^{(\text {tree })}+\mathrm{Cov}^{(\mathrm{SSC})}$.

and

$$
\begin{gathered}
D_{0}=-\frac{\frac{1}{3}(1+f)+\frac{1}{5} f^{2}+\frac{1}{7} f^{3}}{1+\frac{2}{3} f+\frac{1}{5} f^{2}}, \\
D_{2}=-\frac{\frac{2}{3} f+\frac{4}{7} f^{2}+\frac{10}{63} f^{3}}{\frac{4}{3} f+\frac{4}{7} f^{2}},
\end{gathered}
$$

where the linear bias is set to be unity here. The density fluctuation in a given survey is defined against the mean within the survey window rather than the global mean and thereby the normalization of the power spectrum is altered as [64]

$$
P_{\ell}^{\mathrm{w}}(k)=\frac{P_{\ell}(k)}{\left(1+\delta_{\mathrm{b}}^{(\mathrm{z})}\right)^{2}},
$$

where $\delta_{\mathrm{b}}^{(\mathrm{z})}$ is the background density in redshift space and thereby the response is changed to

$\frac{\partial \ln P_{\ell}(k)}{\partial \delta_{\mathrm{b}}} \rightarrow \frac{\partial \ln P_{\ell}^{\mathrm{w}}(k)}{\partial \delta_{\mathrm{b}}} \simeq \frac{\partial \ln P_{\ell}(k)}{\partial \delta_{\mathrm{b}}}-\left(2+\frac{2}{3} f\right)$.

The non-Gaussian covariance is computed as the sum of the tree-level term [Eq. (6)] and the SSC as

$$
\operatorname{Cov}^{(\mathrm{NG})}=\operatorname{Cov}^{(\text {tree })}+\operatorname{Cov}^{(\mathrm{SSC})} .
$$

In Sec. III, we numerically compute the covariance from the ensemble average over $N$-body simulations with the volume of $\left(500 \mathrm{~h}^{-1} \mathrm{Mpc}\right)^{3}$; however, the fluctuations beyond the box size are not taken into account. We compute the covariance including SSC by extracting subboxes with the volume of $\left(500 h^{-1} \mathrm{Mpc}\right)^{3}$ from eight realizations of large simulation boxes with the volume of $\left(4 h^{-1} \mathrm{Gpc}\right)^{3}$ containing $4096^{3}$ particles. The total number of subboxes becomes $8 \times\left(4 h^{-1} \mathrm{Gpc} / 500 h^{-1} \mathrm{Mpc}\right)^{3}=4096$. The mean density field is computed in each subbox and the shift field for reconstruction is computed from the smoothed density field using particle data in each subbox. The reconstructed density field is also computed in each subbox data including mass particles shifted from neighboring subboxes. Strictly speaking, the fluctuation beyond the large simulation box size $4 h^{-1} \mathrm{Gpc}$ is not included in the covariance; however, the SSC is dominated by the fluctuations below this size. Reference [61] also addressed the question of what box size is needed to model the largescale structure and argued that modes larger than $1 h^{-1} \mathrm{Gpc}$ do not contribute. For the purpose of comparison with the perturbation theory, however, we integrate $k$ from $2 \pi /\left(4 h^{-1} \mathrm{Gpc}\right)$ in the calculation of $\sigma_{b}$ [Eq. (21)].

Figure 7 shows the comparison of the $\mathrm{S} / \mathrm{N}$ for prerec and postrec spectra with $R_{s}=10 h^{-1} \mathrm{Mpc}$. We find that both $\mathrm{S} / \mathrm{N}$ decrease when including SSC. Since the reconstruction is performed within the survey area, the bulk motion of supersample modes cannot be corrected by the reconstruction. The postrec spectra, however, have still higher $\mathrm{S} / \mathrm{N}$ than the prerec spectra. The reconstruction improves $\mathrm{S} / \mathrm{N}$ by $5 \%$ $\left(k_{\max }=0.1 \mathrm{~h} / \mathrm{Mpc}\right)$ and by $19 \%\left(k_{\max }=0.2 \mathrm{~h} / \mathrm{Mpc}\right)$ at 

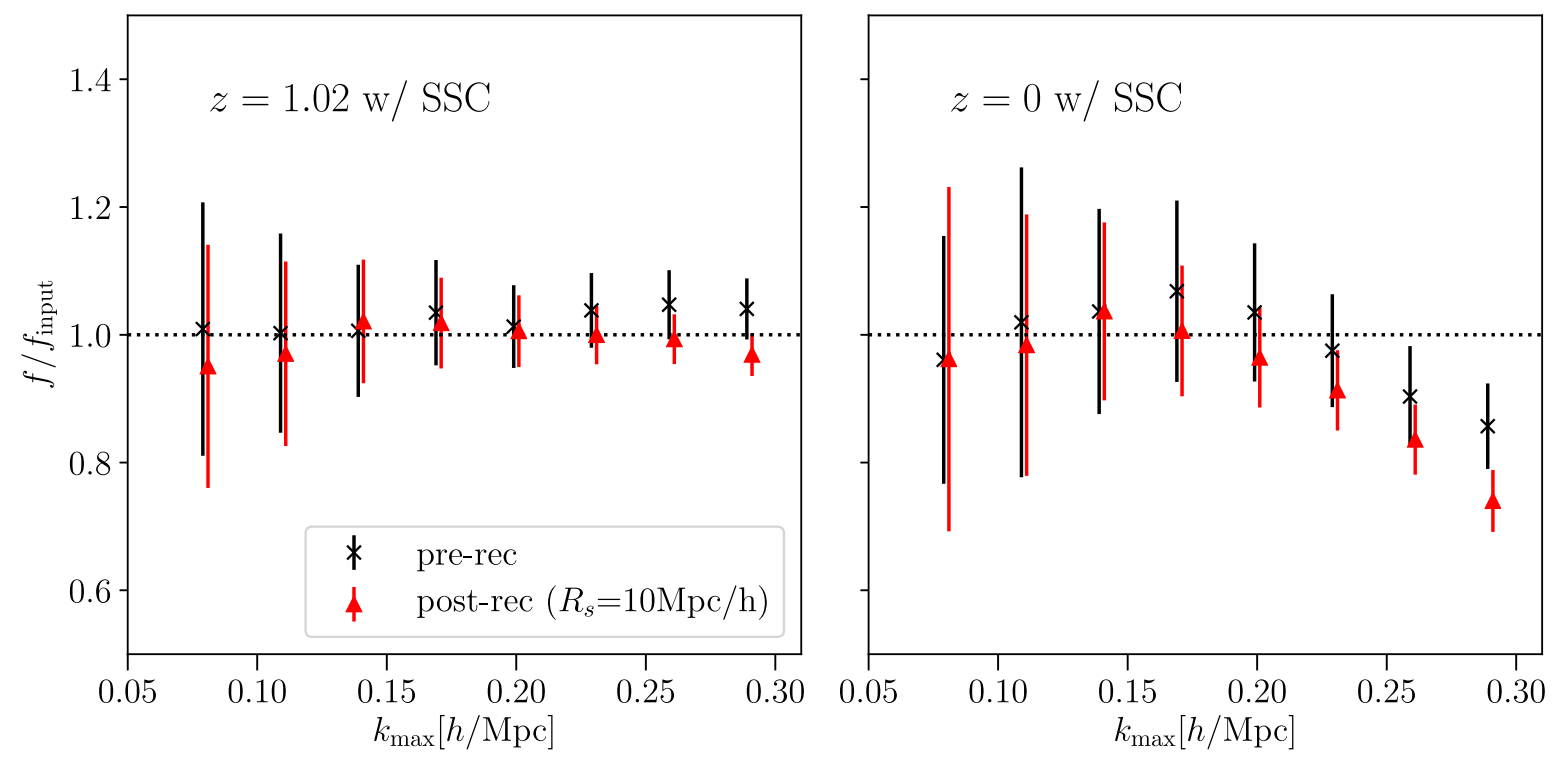

FIG. 8. Same as Fig. 6 but for the SSC effect is included. Here we fix the smoothing scale $R_{s}$ for the postrec spectra $10 h^{-1}$ Mpc.

$z=1.02$ and by $14 \%\left(k_{\max }=0.1 \mathrm{~h} / \mathrm{Mpc}\right)$ and by $40 \%$ $\left(k_{\max }=0.2 \mathrm{~h} / \mathrm{Mpc}\right)$ at $z=0$. The perturbation formulas also show the consistent results with the numerical one and they quantitatively agree up to $k \sim 0.1 \mathrm{~h} / \mathrm{Mpc}$.

Figure 8 shows the impact on the growth rate measurements when including SSC. The input value of growth rate is again recovered up to $k_{\max } \sim 0.3 h / \mathrm{Mpc}$ at $z=1.02$ and $k_{\max } \sim 0.2 \mathrm{~h} / \mathrm{Mpc}$ at $z=0$ for both prerec and postrec spectra. The improvements of the error by the reconstruction with $R_{s}=10 h^{-1} \mathrm{Mpc}$ are $13 \%$ when $k_{\max }=0.2 h / \mathrm{Mpc}$ and $30 \%$ when $k_{\max }=0.3 \mathrm{~h} / \mathrm{Mpc}$ at $z=1.02$ and $28 \%$ when $k_{\max }=0.2 \mathrm{~h} / \mathrm{Mpc}$ at $z=0$, which are comparable to the improvement without SSC.

\section{SUMMARY AND CONCLUSIONS}

We investigated the covariance of the redshift-space matter power spectra after a standard density-field reconstruction that is commonly used in the $\mathrm{BAO}$ analysis. We derived the perturbative formula of the covariance of the multipole components of the power spectra at tree level. We find that the positive off-diagonal components of the covariance from the tree-level trispectra decrease after the reconstruction and have negative values at the smoothing scale of the shift field $R_{s}$ less than $\sim 10 h^{-1} \mathrm{Mpc}$. We also computed the covariance of the multipole power spectra directly from a large set of $N$-body simulations. We find the significant decrease of the off-diagonal components and the behavior is consistent with the perturbation theory. In consequence, the information content of the postrec power spectra evaluated with the $\mathrm{S} / \mathrm{N}$ of their monopole and quadrupole components significantly increase compared to the prerec power spectra. Interestingly, the $\mathrm{S} / \mathrm{N}$ of the reconstructed spectra with $R_{S}$ less than $10 h^{-1}$ Mpc exceeds to that of the linear spectrum with the Gaussian covariance, which comes from the negative off-diagonal components of the covariance matrix. The enhancement of the $\mathrm{S} / \mathrm{N}$ is more significant at later times. We also studied the supersample covariance effect both from perturbative and numerical approaches. We find that the $\mathrm{S} / \mathrm{N}$ reduces even after the reconstruction because the reconstruction performs within the survey area and thus the bulk motion of the supersample modes cannot be corrected by reconstruction. Even when the SSC is included, the postrec spectra still have higher $\mathrm{S} / \mathrm{N}$ than the prerec spectra.

We find that the tree-level perturbative approach is limited to describe the simulated covariance at $k \leq 0.1 \mathrm{~h} / \mathrm{Mpc}$. This indicates that higher-order mode coupling needs to be taken into account to describe the covariance more accurately. There are several works to describe mode couplings at higher $k$ based on the effective field theory [83], the response approach [84], and also semianalytical models [85-87]. It may be interesting to apply these methods to describe the covariance of reconstructed spectra.

Recovery of cosmological information in the two-point statistics makes the cosmological analysis simpler. We demonstrated that the reconstruction significantly reduced the error of growth rate inferred from the redshift-space power spectrum. So far, the reconstruction has been mainly applied to the BAO analysis due to the lack of theoretical understandings of the reconstructed spectrum. Since it is found that the error of the full shape of the power spectrum is improved, it is interesting to investigate how the other cosmological parameters are improved by using the information of the full shape of power spectra after reconstruction. We also have to take into account the galaxy bias and the shot noise as well as various observational effects such as survey geometry to apply the actual observational data (e.g., [70]). The shot noise increases the statistical uncertainties in the power spectrum [Eq. (2)] and also in the shift field for 
reconstruction particularly when the smoothing scale is small. We leave this for future work.

\section{ACKNOWLEDGMENTS}

We thank an anonymous referee for useful comments. This work was supported by MEXT/JSPS KAKENHI Grants No. JP16K17684 (C. H.), No. JP18H04348 (C. H.),
No. JP17H01131 (R. T.), and No. 20H04723 (R. T.). The work of K. K. was supported by the UK STFC Grant No. ST/ S000550/1 and the European Research Council under the European Union's Horizon 2020 programme (Grant No. 646702 "CosTesGrav"). Numerical computations were in part carried out on Cray XC30 and XC50 at Centre for Computational Astrophysics, National Astronomical Observatory of Japan.
[1] L. Amendola, C. Quercellini, and E. Giallongo, Mon. Not. R. Astron. Soc. 357, 429 (2005).

[2] D. J. Eisenstein, W. Hu, and M. Tegmark, Astrophys. J. Lett. 504, L57 (1998).

[3] A. Meiksin, M. White, and J. A. Peacock, Mon. Not. R. Astron. Soc. 304, 851 (1999).

[4] C. Blake and K. Glazebrook, Astrophys. J. 594, 665 (2003).

[5] W. Hu and Z. Haiman, Phys. Rev. D 68, 063004 (2003).

[6] T. Matsubara, Astrophys. J. 615, 573 (2004).

[7] R. E. Angulo, C. M. Baugh, C. S. Frenk, R. G. Bower, A. Jenkins, and S. L. Morris, Mon. Not. R. Astron. Soc. 362, L25 (2005).

[8] H.-J. Seo and D. J. Eisenstein, Astrophys. J. 633, 575 (2005).

[9] M. White, Astropart. Phys. 24, 334 (2005).

[10] D. J. Eisenstein, I. Zehavi, D. W. Hogg, R. Scoccimarro, M. R. Blanton, R. C. Nichol, R. Scranton, H.-J. Seo, M. Tegmark, Z. Zheng et al., Astrophys. J. 633, 560 (2005).

[11] S. Cole, W. J. Percival, J. A. Peacock, P. Norberg, C. M. Baugh, C. S. Frenk, I. Baldry, J. Bland-Hawthorn, T. Bridges, R. Cannon et al., Mon. Not. R. Astron. Soc. 362, 505 (2005).

[12] N. Padmanabhan, D. J. Schlegel, U. Seljak, A. Makarov, N. A. Bahcall, M. R. Blanton, J. Brinkmann, D. J. Eisenstein, D. P. Finkbeiner, J. E. Gunn et al., Mon. Not. R. Astron. Soc. 378, 852 (2007).

[13] W. J. Percival, R. C. Nichol, D. J. Eisenstein, J. A. Frieman, M. Fukugita, J. Loveday, A. C. Pope, D. P. Schneider, A. S. Szalay, M. Tegmark et al., Astrophys. J. 657, 645 (2007).

[14] D. J. Eisenstein, H.-J. Seo, and M. White, Astrophys. J. 664, 660 (2007).

[15] E. Huff, A. E. Schulz, M. White, D. J. Schlegel, and M. S. Warren, Astropart. Phys. 26, 351 (2007).

[16] R. E. Angulo, C. M. Baugh, C. S. Frenk, and C. G. Lacey, Mon. Not. R. Astron. Soc. 383, 755 (2008).

[17] T. Okumura, T. Matsubara, D. J. Eisenstein, I. Kayo, C. Hikage, A. S. Szalay, and D. P. Schneider, Astrophys. J. 676, 889 (2008).

[18] X. Xu, A. J. Cuesta, N. Padmanabhan, D. J. Eisenstein, and C. K. McBride, Mon. Not. R. Astron. Soc. 431, 2834 (2013).

[19] L. Anderson, É. Aubourg, S. Bailey, F. Beutler, V. Bhardwaj, M. Blanton, A. S. Bolton, J. Brinkmann, J. R. Brownstein, A. Burden et al., Mon. Not. R. Astron. Soc. 441, 24 (2014).
[20] R. Tojeiro, A. J. Ross, A. Burden, L. Samushia, M. Manera, W. J. Percival, F. Beutler, J. Brinkmann, J. R. Brownstein, A. J. Cuesta et al., Mon. Not. R. Astron. Soc. 440, 2222 (2014).

[21] E. A. Kazin, J. Koda, C. Blake, N. Padmanabhan, S. Brough, M. Colless, C. Contreras, W. Couch, S. Croom, D. J. Croton et al., Mon. Not. R. Astron. Soc. 441, 3524 (2014).

[22] A. J. Ross, L. Samushia, C. Howlett, W. J. Percival, A. Burden, and M. Manera, Mon. Not. R. Astron. Soc. 449, 835 (2015).

[23] S. Alam, M. Ata, S. Bailey, F. Beutler, D. Bizyaev, J. A. Blazek, A. S. Bolton, J. R. Brownstein, A. Burden, C.-H. Chuang et al., Mon. Not. R. Astron. Soc. 470, 2617 (2017).

[24] F. Beutler, H.-J. Seo, A. J. Ross, P. McDonald, S. Saito, A. S. Bolton, J. R. Brownstein, C.-H. Chuang, A. J. Cuesta, D. J. Eisenstein et al., Mon. Not. R. Astron. Soc. 464, 3409 (2017).

[25] L. Guzzo, M. Pierleoni, B. Meneux, E. Branchini, O. Le Fèvre, C. Marinoni, B. Garilli, J. Blaizot, G. De Lucia, A. Pollo et al., Nature (London) 451, 541 (2008).

[26] K. Yamamoto, T. Sato, and G. Hütsi, Prog. Theor. Phys. 120, 609 (2008).

[27] B. A. Reid, L. Samushia, M. White, W. J. Percival, M. Manera, N. Padmanabhan, A. J. Ross, A. G. Sánchez, S. Bailey, D. Bizyaev et al., Mon. Not. R. Astron. Soc. 426, 2719 (2012).

[28] F. Beutler, S. Saito, H.-J. Seo, J. Brinkmann, K. S. Dawson, D. J. Eisenstein, A. Font-Ribera, S. Ho, C. K. McBride, F. Montesano et al., Mon. Not. R. Astron. Soc. 443, 1065 (2014).

[29] L. Samushia, B. A. Reid, M. White, W. J. Percival, A. J. Cuesta, G.-B. Zhao, A. J. Ross, M. Manera, É. Aubourg, F. Beutler et al., Mon. Not. R. Astron. Soc. 439, 3504 (2014).

[30] A. Oka, S. Saito, T. Nishimichi, A. Taruya, and K. Yamamoto, Mon. Not. R. Astron. Soc. 439, 2515 (2014).

[31] C. Hikage and K. Yamamoto, J. Cosmol. Astropart. Phys. 08 (2013) 019.

[32] M. M. Ivanov, M. Simonović, and M. Zaldarriaga, J. Cosmol. Astropart. Phys. 05 (2020) 042.

[33] M. Takada, R. Ellis, M. Chiba, J. E. Greene, H. Aihara, N. Arimoto, K. Bundy, J. Cohen, O. Doré, G. Graves et al., Publ. Astron. Soc. Jpn. 66, R1 (2014).

[34] A. Aghamousa, J. Aguilar, S. Ahlen, S. Alam, L. E. Allen, C. Allende Prieto, J. Annis, S. Bailey, C. Balland et al. (DESI Collaboration), arXiv:1611.00036. 
[35] G. J. Hill, K. Gebhardt, E. Komatsu, N. Drory, P. J. MacQueen, J. Adams, G. A. Blanc, R. Koehler, M. Rafal, M. M. Roth et al., ASP Conf. Ser. 399, 115 (2008).

[36] L. Amendola, S. Appleby, A. Avgoustidis, D. Bacon, T. Baker, M. Baldi, N. Bartolo, A. Blanchard, C. Bonvin, S. Borgani et al., Living Rev. Relativity 21, 2 (2018).

[37] D. Spergel, N. Gehrels, C. Baltay, D. Bennett, J. Breckinridge, M. Donahue, A. Dressler, B. S. Gaudi, T. Greene, O. Guyon et al., arXiv:1503.03757.

[38] M. Crocce and R. Scoccimarro, Phys. Rev. D 77, 023533 (2008).

[39] R. Scoccimarro, M. Zaldarriaga, and L. Hui, Astrophys. J. 527, 1 (1999).

[40] R. Takahashi, N. Yoshida, M. Takada, T. Matsubara, N. Sugiyama, I. Kayo, A. J. Nishizawa, T. Nishimichi, S. Saito, and A. Taruya, Astrophys. J. 700, 479 (2009).

[41] J. Carron, M. Wolk, and I. Szapudi, Mon. Not. R. Astron. Soc. 453, 450 (2015).

[42] D. J. Eisenstein, H.-J. Seo, E. Sirko, and D. N. Spergel, Astrophys. J. 664, 675 (2007).

[43] Y. B. Zel'dovich, Astron. Astrophys. 5, 84 (1970).

[44] H.-J. Seo, E. R. Siegel, D. J. Eisenstein, and M. White, Astrophys. J. 686, 13 (2008).

[45] N. Padmanabhan, M. White, and J. D. Cohn, Phys. Rev. D 79, 063523 (2009).

[46] Y. Noh, M. White, and N. Padmanabhan, Phys. Rev. D 80, 123501 (2009).

[47] H.-J. Seo, J. Eckel, D. J. Eisenstein, K. Mehta, M. Metchnik, N. Padmanabhan, P. Pinto, R. Takahashi, M. White, and X. $\mathrm{Xu}$, Astrophys. J. 720, 1650 (2010).

[48] B. D. Sherwin and M. Zaldarriaga, Phys. Rev. D 85, 103523 (2012).

[49] N. Padmanabhan, X. Xu, D. J. Eisenstein, R. Scalzo, A. J. Cuesta, K. T. Mehta, and E. Kazin, Mon. Not. R. Astron. Soc. 427, 2132 (2012).

[50] S. Tassev and M. Zaldarriaga, J. Cosmol. Astropart. Phys. 10 (2012) 006.

[51] M. Schmittfull, Y. Feng, F. Beutler, B. Sherwin, and M. Y. Chu, Phys. Rev. D 92, 123522 (2015).

[52] H.-J. Seo, F. Beutler, A. J. Ross, and S. Saito, Mon. Not. R. Astron. Soc. 460, 2453 (2016).

[53] M. Schmittfull, T. Baldauf, and M. Zaldarriaga, Phys. Rev. D 96, 023505 (2017).

[54] X. Wang, H.-R. Yu, H.-M. Zhu, Y. Yu, Q. Pan, and U.-L. Pen, Astrophys. J. Lett. 841, L29 (2017).

[55] Y. Yu, H.-M. Zhu, and U.-L. Pen, Astrophys. J. 847, 110 (2017).

[56] R. Hada and D. J. Eisenstein, Mon. Not. R. Astron. Soc. 478, 1866 (2018).

[57] C. Hikage, K. Koyama, and A. Heavens, Phys. Rev. D 96, 043513 (2017).

[58] C. Hikage, K. Koyama, and R. Takahashi, Phys. Rev. D 101, 043510 (2020).

[59] J. Harnois-Déraps, U.-L. Pen, I. T. Iliev, H. Merz, J. D. Emberson, and V. Desjacques, Mon. Not. R. Astron. Soc. 436, 540 (2013).
[60] L. Blot, P. S. Corasaniti, J. M. Alimi, V. Reverdy, and Y. Rasera, Mon. Not. R. Astron. Soc. 446, 1756 (2015).

[61] A. Klypin and F. Prada, Mon. Not. R. Astron. Soc. 478, 4602 (2018).

[62] F. Villaescusa-Navarro, C. Hahn, E. Massara, A. Banerjee, A. M. Delgado, D. Kodi Ramanah, T. Charnock, E. Giusarma, Y. Li, E. Allys et al., Astrophys. J. Suppl. Ser. 250, 2 (2020).

[63] A. J. S. Hamilton, C. D. Rimes, and R. Scoccimarro, Mon. Not. R. Astron. Soc. 371, 1188 (2006).

[64] M. Takada and W. Hu, Phys. Rev. D 87, 123504 (2013).

[65] Y. Li, W. Hu, and M. Takada, Phys. Rev. D 89, 083519 (2014).

[66] P. A. R. Ade, N. Aghanim, M. Arnaud, M. Ashdown, J. Aumont, C. Baccigalupi, A. J. Banday, R. B. Barreiro, J. G. Bartlett et al. (Planck Collaboration), Astron. Astrophys. 594, A13 (2016).

[67] J. N. Fry, Astrophys. J. 279, 499 (1984).

[68] R. Scoccimarro, H. M. P. Couchman, and J. A. Frieman, Astrophys. J. 517, 531 (1999).

[69] T. Matsubara, Phys. Rev. D 77, 063530 (2008).

[70] D. Wadekar and R. Scoccimarro, arXiv:1910.02914.

[71] V. Springel, Mon. Not. R. Astron. Soc. 364, 1105 (2005).

[72] M. Crocce, S. Pueblas, and R. Scoccimarro, Mon. Not. R. Astron. Soc. 373, 369 (2006).

[73] T. Nishimichi, A. Shirata, A. Taruya, K. Yahata, S. Saito, Y. Suto, R. Takahashi, N. Yoshida, T. Matsubara, N. Sugiyama et al., Publ. Astron. Soc. Jpn. 61, 321 (2009).

[74] T. Nishimichi, M. Takada, R. Takahashi, K. Osato, M. Shirasaki, T. Oogi, H. Miyatake, M. Oguri, R. Murata, Y. Kobayashi et al., Astrophys. J. 884, 29 (2019).

[75] A. Lewis, A. Challinor, and A. Lasenby, Astrophys. J. 538, 473 (2000).

[76] Note1, fFTW3 at http://www.fftw.org.

[77] Y. P. Jing, Astrophys. J. 620, 559 (2005).

[78] J. Hartlap, P. Simon, and P. Schneider, Astron. Astrophys. 464, 399 (2007).

[79] J. J. M. Carrasco, M. P. Hertzberg, and L. Senatore, J. High Energy Phys. 09 (2012) 082.

[80] F. Feroz, M. P. Hobson, and M. Bridges, Mon. Not. R. Astron. Soc. 398, 1601 (2009).

[81] B. Audren, J. Lesgourgues, K. Benabed, and S. Prunet, J. Cosmol. Astropart. Phys. 02 (2013) 001.

[82] Y. Li, M. Schmittfull, and U. Seljak, J. Cosmol. Astropart. Phys. 02 (2018) 022.

[83] D. Bertolini, K. Schutz, M. P. Solon, J. R. Walsh, and K. M. Zurek, Phys. Rev. D 93, 123505 (2016).

[84] A. Barreira and F. Schmidt, J. Cosmol. Astropart. Phys. 11 (2017) 051.

[85] M. C. Neyrinck, Astrophys. J. 736, 8 (2011).

[86] I. Mohammed and U. Seljak, Mon. Not. R. Astron. Soc. 445, 3382 (2014).

[87] I. Mohammed, U. Seljak, and Z. Vlah, Mon. Not. R. Astron. Soc. 466, 780 (2017). 